\section{STRUCTURAL}

\section{BIOLOGY}

ISSN 2059-7983

\title{
Sir Aaron Klug OM FRS (1926-2018)
}

\author{
R. A. Crowther*
}

MRC Laboratory of Molecular Biology, Cambridge, UK. *Correspondence e-mail: rac1@mrc-Imb.cam.ac.uk

Aaron Klug died in Cambridge on 20 November 2018. He contributed hugely to the development of structural molecular biology, where his interests spanned a wide range of topics, to each of which he made substantial contributions. Starting with X-ray diffraction of crystals and helical viruses, he moved on to electron microscopy, where he initiated methods for the quantitative interpretation of micrographs and particularly for making three-dimensional maps. He applied these techniques to a wide range of biological assemblies, including viruses, chromatin, nucleic acids and zinc fingers.

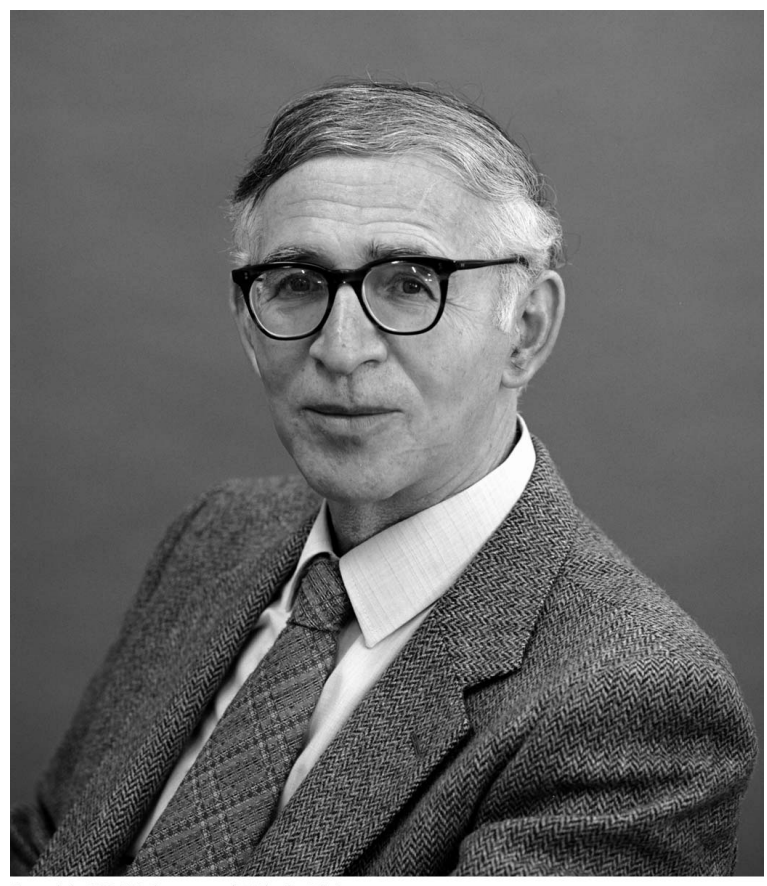

Copyright MRC Laboratory of Molcular Biology

Aaron was born in Lithuania but, when he was two, the family fled to South Africa, where they lived in Durban. He attended Durban High School and made rapid progress with his studies. Among many other books, he read The Microbe Hunters by Paul de Kruif and became fascinated by microbiology. This prompted him to take courses in medicine when, aged just 15 , he joined the University of Witwatersrand in Johannesburg. However he did not enjoy the medical courses and switched to physical sciences, taking chemistry, physics and mathematics. Having graduated he moved to Cape Town to do a Master's Degree in physics and worked on X-ray crystallography with R. W. James. He learned much on the topic by helping to check the proofs of James's book on The Optical Principles of the Diffraction of X-rays. In 1949 he won an 1851 scholarship to pursue a $\mathrm{PhD}$ at Trinity College, Cambridge. He had hoped to join the new MRC Unit directed by Max Perutz and to work on the structure of proteins. However Sir Lawrence Bragg, Director of The Cavendish Laboratory, assigned him to a theoretical project on the microstructure of cooling steels.

The post-doctoral award of a Nuffield Fellowship enabled him to move to Birkbeck College, London, initially to work on X-ray crystallography of ribonuclease. It was here that he met Rosalind Franklin and, when the ribonuclease project failed to make progress, they decided to work together on the structure of viruses. Rosalind was using $\mathrm{X}$-ray diffraction to analyse fibres of the rod-shaped tobacco mosaic virus and crystals of 
spherical viruses. The X-ray patterns from TMV prompted him to develop further the theory of diffraction from helical structures. The work on icosahedral viruses led to the classic paper with Donald Caspar on the physical principles in the construction of regular viruses, which introduced the idea of quasi-equivalent packing of protein molecules.

After Franklin's death in 1958, Aaron took over her group and they moved in 1962 to the newly opened MRC Laboratory of Molecular Biology in Cambridge. Here, with John Finch, he initiated a quantitative analysis of electron micrographs, which had hitherto been viewed qualitatively. The first step was to use the micrograph as a diffraction grating to display the Fourier components in the image, using an optical diffractometer developed for X-ray crystal analysis. This led to the idea of spatial Fourier filtering to separate the layers in the image of a multilayer periodic specimen. However, the separated images still represented projections of the scattering matter in each layer. This prompted the development with David DeRosier of a method which they termed threedimensional reconstruction, published in 1968, and based on the relationship well known to crystallographers between the projection of a structure and the corresponding central section of the three-dimensional Fourier transform. Initially it was applied to make three-dimensional maps at low resolution of the then available negatively stained specimens. Latterly, with the development of cryo-microscopy of frozen hydrated specimens, it has become a powerful technique to reveal the atomic details of many important biological molecules.

With collaborators Aaron's later crystallographic studies encompassed the disks of TMV coat protein, yeast phenylalanine tRNA and a self-cleaving ribozyme, the nucleosome particle of chromatin and various constructs containing zinc fingers, a DNA-binding motif that he had discovered in transcription factors. He was also instrumental in starting a project to analyse the molecular nature of paired helical filaments that form intracellular tangles in the brain in Alzheimer's disease.

Aaron received many honours, including the 1982 Nobel Prize in chemistry. He was Director of the Laboratory of Molecular Biology from 1986 to 1996, during which time he helped to establish the Sanger Centre, now the Wellcome Sanger Institute. He was President of the Royal Society of London from 1995 to 2000, where he made important contributions to the public understanding of science. He was one of the great innovators in the growth of structural molecular biology and will be much missed. 